\title{
Preparing for the Impact of the ICD-9/10 Transition on Syndromic Surveillance
}

\author{
Peter Hicks ${ }^{1}$, Julie A. Pavlin², Atar Baer ${ }^{3}$, David Swenson4, Aaron Kite-Powell ${ }^{5}$, Achala U. \\ Jayatilleke ${ }^{1}$, Brooke Evans ${ }^{\star 6}$ and Laura C. Streichert ${ }^{6}$ \\ ${ }^{1} \mathrm{CDC}$, Atlanta, GA, USA; ${ }^{2}$ Armed Forces Health Surveillance Center, Silver Spring, MD, USA; ${ }^{3}$ Public Health—Seattle \& King County, \\ Seattle, WA, USA; ${ }^{4}$ State of New Hampshire, Concord, NH, USA; ${ }^{5}$ CNTS support to AFHSC, Silver Spring, MD, USA; ${ }^{6}$ ISDS, Brighton, \\ MA, USA
}

\section{Objective}

To describe the process undertaken to translate syndromic surveillance syndromes and sub-syndromes from ICD-9 diagnostic codes to ICD-10 codes and how these translations can be used to improve syndromic surveillance practice.

\section{Introduction}

The US Department of Health and Human Services has mandated that after October 1, 2015, all HIPAA covered entities must transition from using International Classification of Diseases version 9 (ICD9) codes to using version 10 (ICD-10) codes (www.cms.gov). This will impact public health surveillance entities that receive, analyze, and report ICD-9 encoded data. Public health agencies will need to modify existing database structures, extraction rules, and messaging guides, as well as syndrome definitions and underlying analytics, statistical methodologies, and business rules. Implementation challenges include resources, funding, workforce capabilities, and time constraints for code translation and syndrome reclassification.

To address these challenges, the International Society for Disease Surveillance (ISDS), in partnership with the CDC and the Council of State and Territorial Epidemiologists (CSTE), conducted a project to develop consensus-driven syndrome definitions based on ICD10 codes. Over 130 newly created ICD-9 to-ICD-10 mappings and corresponding syndromic definitions were fully reviewed and vetted by the syndromic surveillance community, which relies on these codes for routine surveillance and research purposes. The final mappings may be leveraged by other federal, state, and local public health entities to better prepare and improve their surveillance, analytics, and reporting activities impacted by the ICD-10 transition.

\section{Methods}

Translation from ICD-9 to ICD-10 codes involves a rethinking of how syndromes and sub-syndromes are defined and aligned with practical syndromic surveillance practice. To address this, ISDS coordinated a multi-stakeholder working group to revisit over 130 existing syndromic surveillance definitions and to compile ICD-9 codes that map to these syndromes and sub-syndromes. The individual ICD-9 codes were mapped to ICD-10 codes using freely available General Equivalence Mappings (GEMs). Subsequently, we followed a reverse translation validation process to ensure that the appropriate codes were correctly identified. The resulting Mapping Reference Table (MRT) relates syndromic classifications to both code groupings. These syndrome and sub-syndrome base code groupings were then reviewed by the surveillance community and partner agencies, leveraging clinical and epidemiological expertise, to reach consensus on the final syndrome definitions and mappings.

\section{Results}

The community vetting process demonstrated that a simple translation using GEMs is not adequate and underscored the value of the end-user review. In addition to creating a new ICD-10 resource, the MRT enables users to address challenges associated with changes in baseline trends as a result of the transition. Leveraging the MRT, jurisdictions can quickly map forwards and backwards across the two coding systems to ensure continuity of analytics and reporting during the transition period (Figure 1).

\section{Conclusions}

The higher level of detail inherent to ICD-10 codes will improve the specificity of syndromic surveillance. The development of a consensus-driven MRT will be extremely valuable to entities with the complex task of translating ICD-9 to ICD-10 codes. The code translations will also help the syndromic surveillance community work towards developing standardized syndrome definitions. Future collaborations are under development to re-purpose these code mappings to develop consensus-driven syndromic surveillance definitions that are also vetted though a syndromic surveillance community of practice.

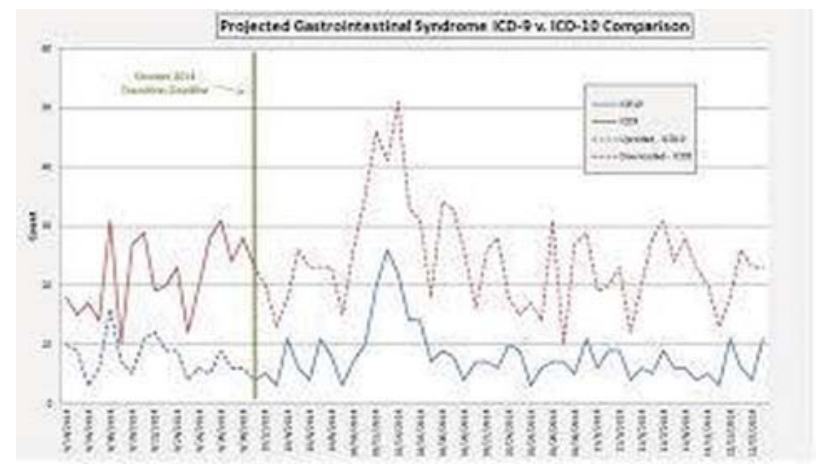

Fig. 1: Forward- and reverse-mapping of ICD 9/10 codes can be helpful for creating a time series that spans the transition. (Hicks, 2013 ISDS Conference).

\section{Keywords}

ICD-10; code mapping; syndromic surveillance; BioSense

\section{Acknowledgments}

We thank the surveillance professionals that assisted with the code set review. This work was supported by the CDC.

\section{*Brooke Evans}

E-mail: bevans@syndromic.org 